\title{
Роль структурных несовершенств графена в резонансном туннелировании через локализованные состояния в $h$-BN барьере ван-дер-ваальсовых гетероструктур
}

\author{
(C) М.В. Григорьев ${ }^{1}$, Д.А. Казарян ${ }^{2,3}$, Е.Е. Вдовин ${ }^{1,1}$, Ю.Н. Ханин ${ }^{1}$, С.В. Морозов ${ }^{1}$, К.С. Новоселов ${ }^{2,4}$ \\ ${ }^{1}$ Институт проблем технологии микроэлектроники и особо чистых материалов Российской академии наук, \\ 142432 Московская область, Черноголовка, Россия \\ ${ }^{2}$ School of Physics and Astronomy, University of Manchester, \\ M13 9PL Manchester, United Kingdom \\ ${ }^{3}$ Department of Physics, National Research University Higher School of Economics, \\ 105066 Moscow, Russia \\ ${ }^{4}$ Department of Material Science \& Engineering, National University of Singapore, \\ 117575 Singapore, Republic of Singapore \\ I E-mail: vdov62@yandex.ru
}

Поступила в Редакцию 26 августа 2019 г.

В окончательной редакции 6 ноября 2019 г.

Принята к публикации 7 ноября 2019 г.

Исследовано резонансное туннелирование через уровни дефектов в $h$-BN барьере ван-дер-ваальсовых гетероструктур. Обнаружен эффект мультиплицирования туннельных резонансов через эти уровни, обусловленный влиянием высокой дефектности структуры соседнего слоя графена, созданной нами преднамеренно с помощью его обработки в плазме. Обсуждены различные механизмы такого влияния.

Ключевые слова: графен, ван-дер-ваальсовы гетероструктуры, дефекты кристаллической решетки, нитрид бора, туннельный транзистор, резонансное туннелирование.

DOI: 10.21883/FTP.2020.03.49025.9249

Ван-дер-ваальсовы гетероструктуры на основе графена и гексагонального нитрида бора $(h$-BN) привлекли в последние годы большое внимание как в фундаментальной физике, так и в прикладных исследованиях. Особая роль гексагонального нитрида бора $(h-\mathrm{BN})$ в таких структурах обусловлена тем, что он обладает большой шириной запрещенной зоны $\sim 5.9$ эВ, а его постоянная решетки только на $1.8 \%$ больше, чем у графена, в результате чего $h$-BN оказался одним из самых актуальных материалов на роль подзатворного диэлектрика или туннельного барьера. Однако недавние спектроскопические исследования электронных и фононных свойств $h$-BN продемонстрировали сильное влияние на эти свойства дефектных состояний в $h$-BN. Так, например, было обнаружено, что множественные (более 50) локализованные состояния дефектов с различными энергиями в пределах его запрещенной зоны порождают туннельные каналы в $h$-BN барьере, которые могут существенно менять электронный транспорт в таких системах, а также приводить к утечкам, телеграфному шуму и пробою подзатворных диэлектриков, и поэтому, естественно, требуют интенсивных дальнейших исследований, так как еще не выяснена однозначно физическая природа их происхождения и причины их неожиданного многообразия [1-6]. К настоящему моменту из общих соображений и простых модельных расчетов предполагается, что локализованные электронные состояния в запрещенной зоне кристаллов $h$-BN могут существовать из-за наличия собственных структурных дефектов и примесей, т.е. вакансий различного типа и атомов внедрения (чаще всего углерода), которые присутствуют даже в номинально чистом кристалле $h$-BN. Кроме того, дефекты могут быть введены непреднамеренно в процессе механического расслоения $h$-BN и его встраивания в многослойную ван-дер-ваальсову гетероструктуру или при других технологических операциях во время создания вертикального графенового транзистора. Но все эти модельные теоретические предположения предсказывают не более десятка различных по энергии уровней локализованных состояний и не объясняют, почему наблюдается такое большое их число в запрещенной зоне $h$-BN экспериментально.

При этом, однако, ранее совершенно не учитывалось возможное влияние на туннелирование через дефекты в $h$-BN барьере структурных несовершенств графеновых контактных слоев, из которых происходит туннелирование, которое может мультиплицировать туннельные резонансы через однотипные уровни дефектов $h$-BN в результате влияния случайных флуктуаций потенциала в контактных слоях, когда энергетический уровень дефектов одного типа с определенной энергией связи проявляется в виде нескольких пиков в токе при разных резонансных напряжениях из-за варьирования порогов туннелирования флуктуациями потенциала. Такой эффект был продемонстрирован при исследовании туннелирования через примесные уровни в $\mathrm{GaAs} / \mathrm{AlAs}-$ гетероструктурах $[7,8]$, где наблюдались множественные резонансные пики при туннелировании через однотипные уровни доноров $\mathrm{Si}$, отражающие флуктуации дна зоны проводимости, индуцированные случайными 
флуктуациями потенциала на границе между высоколегированнной контактной и нелегированной областями GaAs. На основании этих работ мы предположили, что множественные резонансы уровней дефектов $h$-BN могут быть результатом аналогичного влияния случайного потенциала дефектной структуры графена. Для проверки этой гипотезы мы преднамеренно с помощью плазменной обработки вводили большое количество дефектов в верхний графеновый слой туннельной ван-дерваальсовой гетероструктуры и изучили их влияние на туннельный транспорт.

В данной работе исследовался туннельный транспорт в гетероструктурах графен/ $h$-BN/графен с высокой степенью разориентации графеновых слоев и различной степенью совершенства слоев $h$-BN. Это позволило нам в рамках одного эксперимента систематически исследовать проявления дефектных состояний в туннелировании от ситуации их полного отсутствия (в совершенной структуре) до нескольких десятков (после обработки плазмой) в запрещенной зоне $h$-BN и их связи с характеристическими зависимостями электростатической модели идеальной бездефектной гетероструктуры. Было обнаружено предполагавшееся нами мультиплицирование резонансных пиков туннельной проводимости, отражающих переходы через уровни дефектов $h$-BN, вследствие влияния случайного потенциала заряженных вакансий графена, созданных плазменной обработкой структуры. Таким образом, экспериментально показано, что ранее наблюдавшееся и оставшееся необъясненным в работе [5] многообразие дефектных резонансов может быть связано в основном с влиянием случайных флуктуаций потенциала в контактных слоях графена.

Исследовавшиеся образцы представляли собой вертикальные ван-дер-ваальсовы гетероструктуры, получаемые путем механического расслоения и переноса слоев графена и $h$-BN (см. рис. 1). Изучалось два типа образцов, отличавшихся устройством нижнего затвора: в первом типе затвор состоял из высоколегированного $\mathrm{Si}$ и диэлектрических слоев толщиной $D=290 \mathrm{нм}$ $\left(\mathrm{SiO}_{2}\right)+30$ нм $(h$-BN), а во втором - из графенового электрода и слоя $h$-BN толщиной $D=25-30$ нм, что обусловливало разные рабочие интервалы затворных напряжений. Контакты к слоям графена и верхняя затворная металлизация были изготовлены с помощью электронной литографии и напыления Ti/Au. Проводящие графеновые слои были изготовлены в виде крестообразной структуры, разделенной в месте пересечения туннельным барьером $h$-BN толщиной $d$ в несколько (3-6) монослоев, площадь пересечения графеновых электродов составляла 25-50 мкм ${ }^{2}$. Один из образцов преднамеренно подвергался обработке холодной водородной плазмой для внесения множественных дефектов в верхний слой графена. Использовалась плазма постоянного тока в смеси водорода и аргона низкого давления (0.1 мбар при 10\% $\left.\mathrm{H}_{2}\right)$. Измерения туннельной проводимости/тока производились четырехзондовым методом.

Схематическое изображение основных слоев структуры, измерительной цепи и представление основных

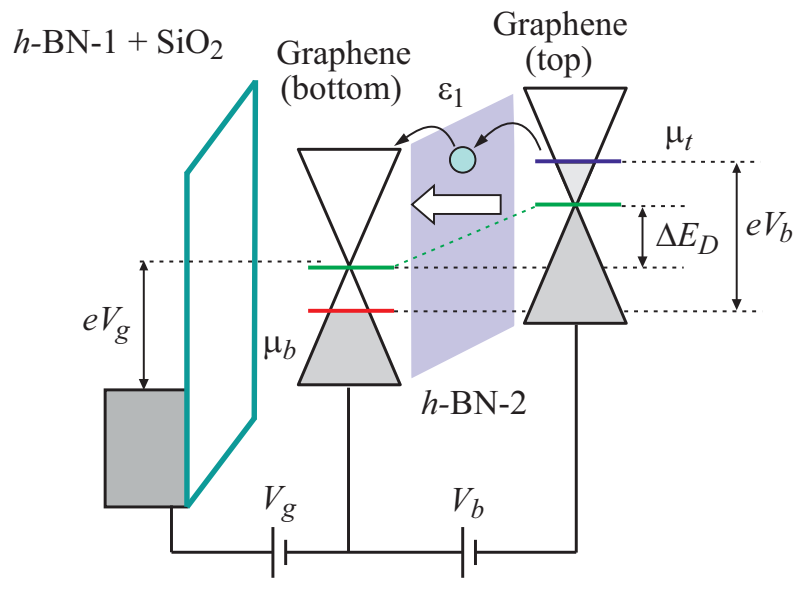

Рис. 1. Схема экспериментальной однозатворной структуры графен/h-BN/графен в электростатической модели конденсатора с тремя обкладками и процессов туннелирования в ней.

туннельных процессов показаны на рис. 1. Несколько атомных слоев $h$-BN образуют туннельный барьер, расположенный между двумя монослоями графена, изображенными в виде сечения конусов, которые действуют как электроды истока и стока. При приложении напряжения смещения, $V_{b}$, между ними химические потенциалы в верхнем и нижнем электродах $\left(\mu_{t}\right.$ и $\left.\mu_{b}\right)$ смещаются друг относительно друга и возникает экспоненциально растущий туннельный ток $I$ через барьер (белая стрелка), как результат переходов носителей из заполненных состояний в одном графеновом электроде в пустые состояния в другом, представленный, например, пунктирной линией на рис. 2, $a$. В случае, если в барьерном слое находится локализованное состояние любой природы и его энергетический уровень, двигаясь с изменением $V_{b}$, совпадает с химическим потенциалом $\mu_{t}$ в верхнем (правом на схеме) контакте, открывается канал резонансного туннелирования через это состояние (черные стрелки), проявляющийся в токе в виде ступени (пика в дифференциальной проводимости), так как при дальнейшем увеличения $V_{b}$ после порога этого резонанса ток через него изменяется относительно слабо (сплошная линия на рис. 2,a). Отметим, что обычно в гетероструктурах большой площади в латеральной плоскости барьера может находиться достаточно много дефектов с одной и той же энергией связи (т.е. одним и тем же порогом туннелирования) и наблюдаемая ступенька в токе будет представлять собой сумму вкладов в ток всех таких однотипных дефектов. В наших образцах кристаллические решетки графеновых электродов были развернуты друг относительно друга на угол больше $10^{\circ}$. Такая разориентация подавляет прямое межзонное резонансное туннелирование с сохранением продольной слоям компоненты импульса и позволяет детально изучить малый туннельный ток при переходах через индивидуальное локализованное состояние [9]. Нижний затворный электрод, расположенный на подложке, ис- 

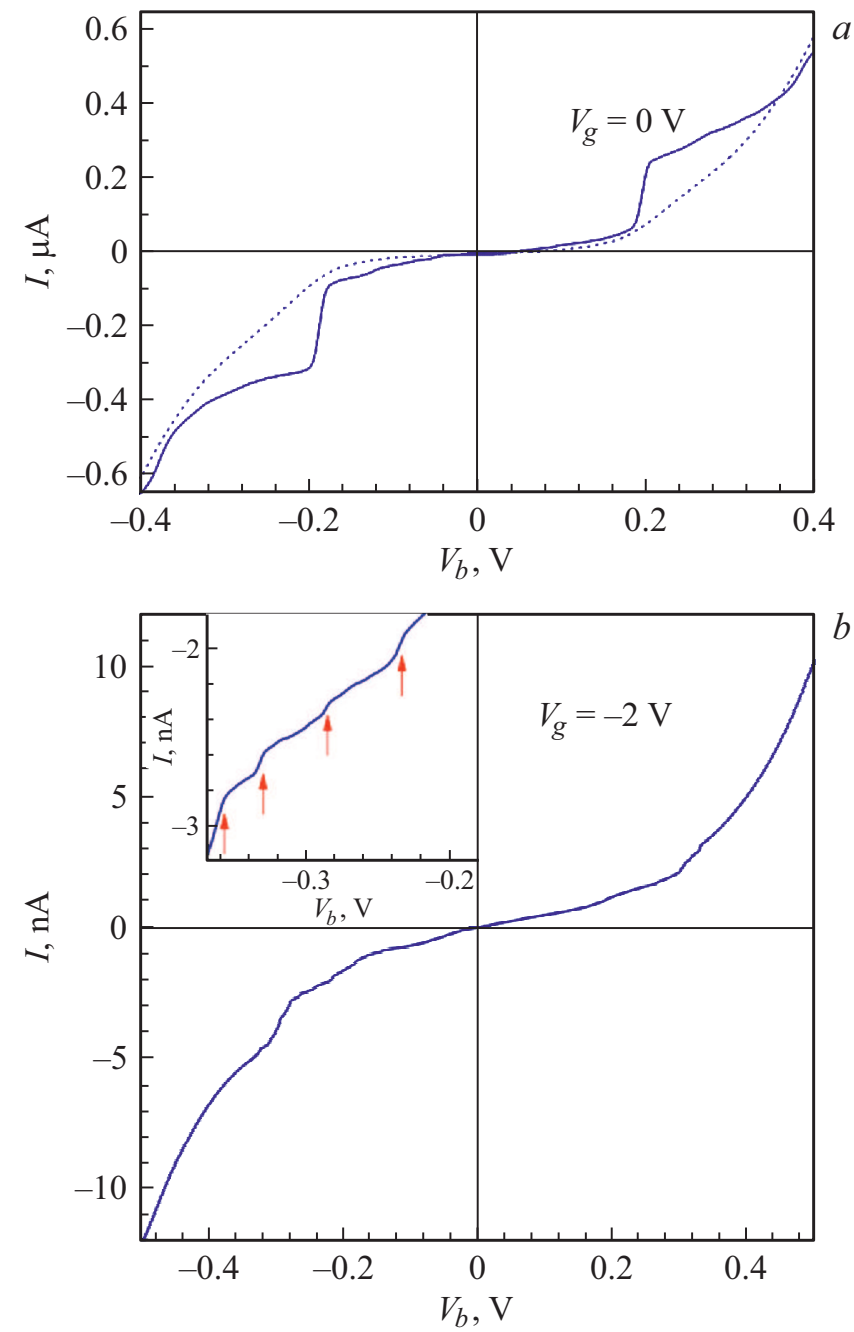

Рис. 2. $a-\mathrm{BAX}$ со ступенькой в токе, обусловленной туннелированием через один уровень дефекта в баьере при $V_{g}=0$ и температуре $T=1.75 \mathrm{~K}$. Пунктирной линией показана ВАХ бездефектного образца с теми же номинальными параметрами структуры при $V_{\text {gate }}=0 ; b-\mathrm{BAX}$ образца при $V_{g}=-2 \mathrm{~B}, T=2 \mathrm{~K}$ с несколькими порогами резонансов. На вставке показана увеличенная область кривой, где стрелками отмечены пороги туннелирования через уровни дефектов.

пользуется для регулировки концентрации носителей в графеновых слоях путем изменения напряжения затвора, $V_{g}$. Энергетический сдвиг дираковских точек, зависящий от $V_{g}$ и $V_{b}$, обозначен на схеме как $\Delta E_{D}$ и для наших образцов равен нулю при $V_{g}$ и $V_{b}=0$ (т.е. в графеновых электродах отсутствует химическое легирование).

В качестве отправной точки нашего эксперимента были исследованы образцы, не подвергавшиеся плазменной обработке. Как и в предыдущих работах, большая часть исследованных образцов демонстрировала монотонные экспоненциальные вольт-амперные характеристики (BAX), обусловленные туннельными переходами электронов из заполненных в пустые состояния дираковских конусов в верхнем и нижнем графеновых слоях без сохранения компоненты импульса вдоль слоев $[9,10]$, свидетельствуя об отсутствии дополнительных каналов туннелирования и, соответственно, дефектов в $h$-BN (пунктирная линия на рис. 2,a). Часть образцов обнаруживала дополнительные ступенчатые особенности на фоне экспоненциальной BAX (см. рис. 2 и вставку на нем), которые обусловлены туннельными переходами через локализованные состояния, аналогично прежним исследованиям полупроводниковых и графеновых систем [1-5]. Положения таких ступенек по $V_{b}$ было случайным на разных образцах, но воспроизводилось для каждого конкретного образца после множественных циклов нагрева и охлаждения. Происхождение таких резонансов обычно связывают с различными видами дефектов в $h$-BN и зависимостью их энергий от положения в различных монослоях барьера.

Наглядное представление о разных режимах транспорта в наших экспериментальных структурах дает рис. 3, на котором показана карта проводимости в координатах $\left(V_{g}, V_{b}\right)$ и характерные сечения этой карты при разных напряжениях $V_{g}$. На рис. 3, $a$ представлена карта проводимости совершенно бездефектного образца. Красная и черная пунктирные кривые отражают расчетные траектории движения моментов пересечения химических потенциалов в верхнем и нижнем слоях $\mu_{t}$
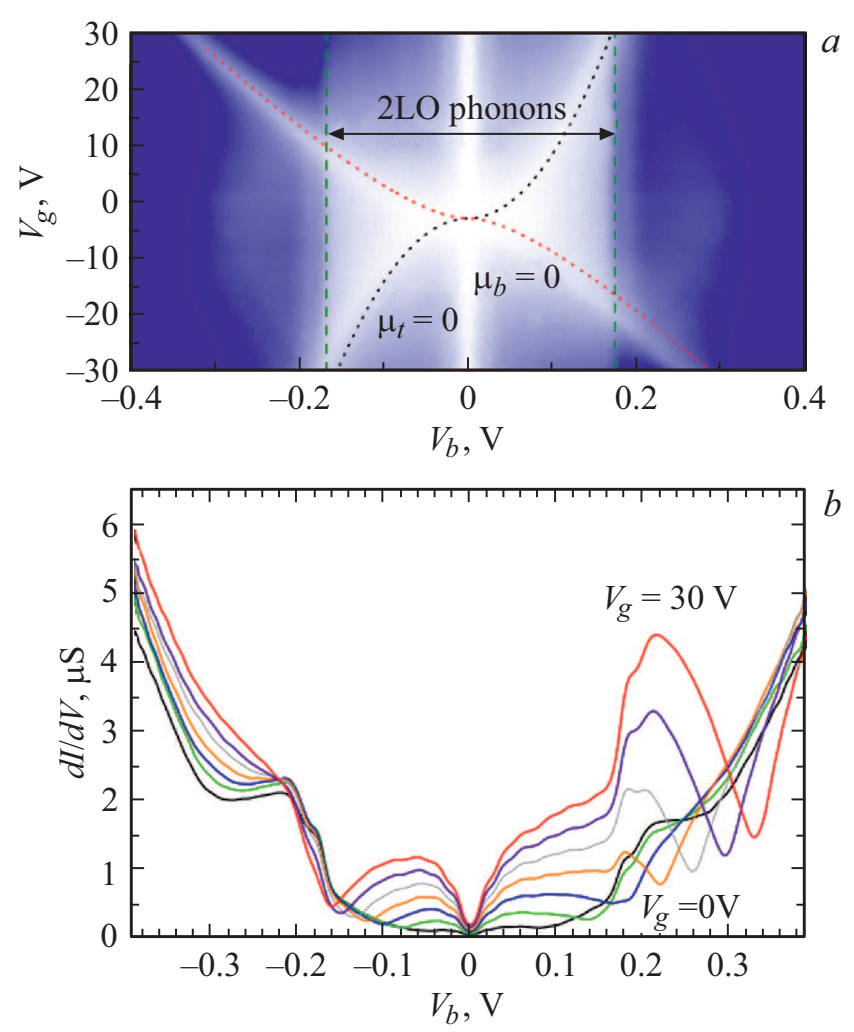

Рис. 3. $a-$ карта проводимости бездефектного образца. Красная и черная пунктирные кривые - расчетные траектории движения моментов пересечения химических потенциалов в верхнем и нижнем электродах $\left(\mu_{t}\right.$ и $\left.\mu_{b}\right)$ с точками электронейтральности; $b-$ характерные зависимости проводимости от смещения, т. е. сечения карты $(a)$ при разных напряжениях $V_{g}$ от 0 до $30 \mathrm{~B}$ с шагом $5 \mathrm{~B}$. 
и $\mu_{b}$ с точками электронейтральности в координатах $V_{g}$ и $V_{g}$, получаемые в соответствии с электростатической моделью идеальной (без учета дефектов $h$-BN) вандер-ваальсовой системы [9-11], которые должны соответствовать минимумам проводимости. Эти характеристические базисные кривые точно определенны для каждого конкретного образца с заданными толщинами диэлектрических слоев $D$ и $d$ и являются основой для дальнейшей идентификации любых других особенностей карт туннельной проводимости. На карте видно хорошее соответствие расчета для образца с толщинами диэлектрических слоев $D=320$ нм и $d=1.5 \mathrm{HM,} \mathrm{ко-}$ торые совпадают с описанными выше номинальными технологическими параметрами, и эксперимента. Прямые вертикальные линии при $V_{\text {bias }} \sim 0.17$ В описывают порог туннелирования с участием оптических фононов, узкая вертикальная полоса возле 0 В отражает область до порога туннелирования с участием акустических фононов [12].

На рис. 4, $а$ показана карта образца с большим $(\sim 10)$ количеством дефектных резонансов в туннельном барьере $h$-BN, BAX которого при $V_{g}=-2$ В рассмотрена выше (см. рис. 2, $b$ ). Такое количество резонансов близко предсказанному теоретически пределу возможных дефектных уровней в $h$-BN. Как видно из карты, почти параллельно базисной характеристической кривой $\mu_{t}=0$, наблюдаются множественные траектории (темные узкие линии максимумов проводимости), соответствующие порогам туннельных переходов через уровни дефектов, когда они совпадают с химическим потенциалом $\mu_{t}$ в верхнем контакте. Различный масштаб затворных напряжений на рис. 3, $a$ и 4, $a$ обусловлен различными толщинами подзатворных диэлектриков в образцах и не влияет на качественное рассмотрение наблюдаемых эффектов.

Далее, для проверки нашего предположения о мультиплицировании резонансов мы подвергли плазменной обработке структуру, которая изначально демонстрировала около десятка резонансов через уровни дефектов, как, например, показано на рис. 4, $a$ и было зарегистрировано в работе [5]. После первоначальных измерений туннельного и латерального транспорта, показавшего малое колличества дефектов в барьере и в контактных слоях графена, образец подвергся воздействию холодной водородной плазмы. Мы использовали плазму постоянного тока в смеси водорода и аргона низкого давления (0.1 мбар при $\left.10 \% \mathrm{H}_{2}\right)$. Образец находился на расстоянии $30 \mathrm{~cm}$ от зоны разряда, чтобы минимизировать возможное повреждение энергичными ионами, так же как в работе [13]. Как обычно, потребовалось около 2 ч плазменной обработки для достижения насыщения измеренных туннельных характеристик. В качестве эталона использовались образцы графена, подвергнутые воздействию чистой плазмы Ar в тех же условиях, которые показали небольшие изменения в их транспортных и рамановских свойствах. Гидрогенизация верхнего открытого графенового слоя в результате обработки в плазме,
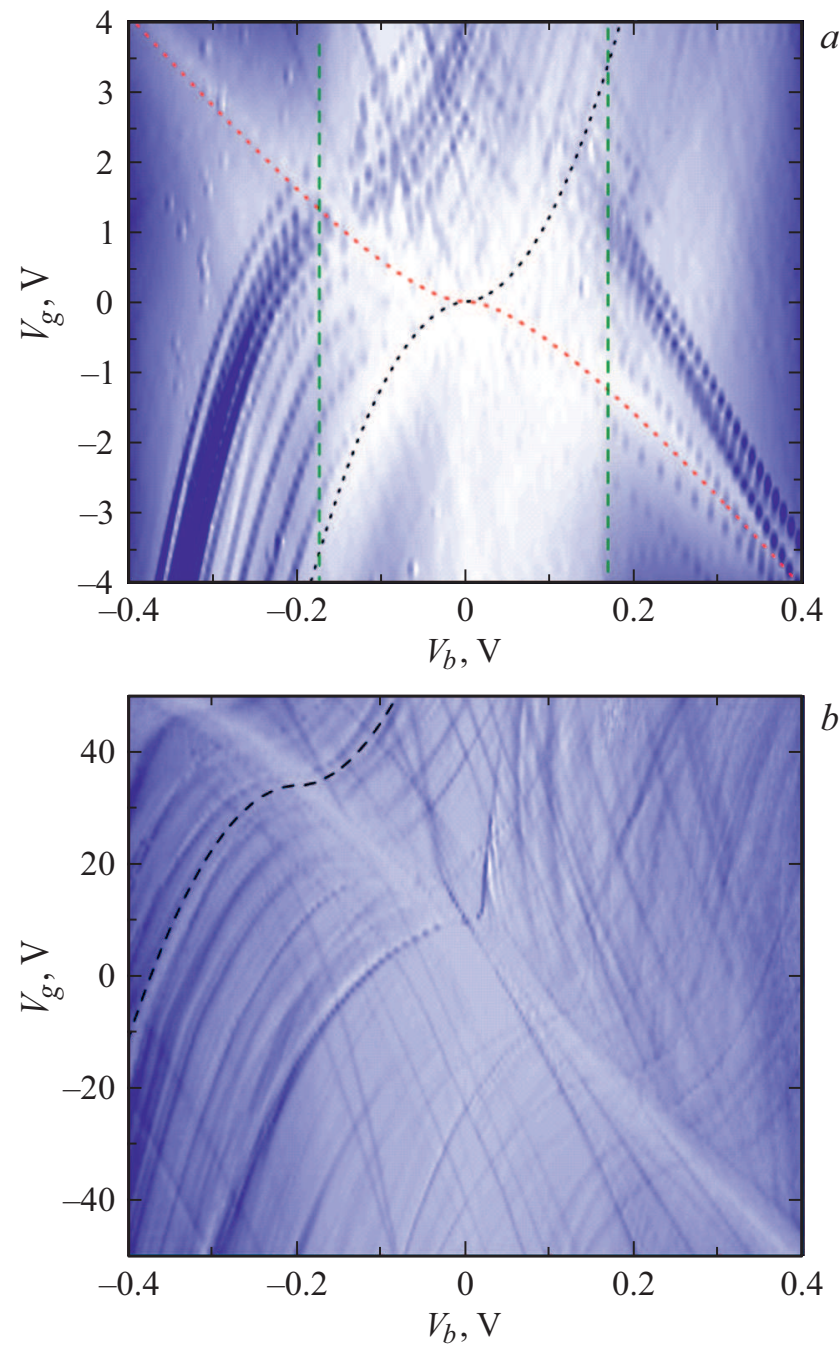

Рис. 4. $a-$ карта образца с большим количеством $(\sim 10)$ дефектных состояний без обработки плазмы. Темно-синиие линии отражают движение порогов резонансов через уровни дефектов; $b$ - карта с мультиплицированным количеством $(\sim 50)$ дефектных состояний после обработки плазмой, черной пунктирной линией показана расчетная функциональная зависимость характеристической линии электростатической модели бездефектного образца.

так же как и в работе [13], была стабильной при комнатной температуре в течение многих дней и образец демонстрировал одинаковые характеристики при повторных измерениях. Обработка плазмой приводила к сдвигу точки электронейтральности на зависимости сопротивления верхнего графена от напряжения на затворе от $V_{g} \approx 0 \mathrm{~B}$ к $V_{g} \approx+50 \mathrm{~B}$, что означает легирование графена дырками с концентрацией $\approx 3 \cdot 10^{12} \mathrm{~cm}^{-2}$ (вероятно, из-за адсорбированной воды). Затем образец отжигался. После отжига (при $450^{\circ} \mathrm{C}$ в атмосфере $\mathrm{Ar}$ в течение 24 ч; более высокая температура обычно повреждает графен) образец вернулся в качественно то же состояние, что и до гидрирования, но со сдвигом точки электронейтральности к $V_{g} \approx+18 \mathrm{~B}$. Такое положение точки элек- 
тронейтральности соответствует наличию легирования графена дырками с концентрацией $n \approx 1 \cdot 10^{12} \mathrm{~cm}^{-2}$. Это связано с появлением множества вакансий в верхнем графеновом слое (со средним интервалом между ними $\approx 10$ нм), вызванным повреждением плазмой, или остаточным кислородом во время отжига [13].

В результате, как видно из рис. $4, b$, на карте проводимости появились множественные $(\sim 50)$ траектории, поведение которых повторяет вид расчетной функциональной зависимости характеристической линии электростатической модели бездефектного образца, показанной черной пунктирной линией на рис. 4, $b$. Это однозначно указывает на то, что появившиеся линии, как и ранее, соответствуют порогам резонансов через уровни дефектов в барьере. Для качественного объяснения причин появления после плазменной обработки множества новых резонансных траекторий на карте проводимости, параллельных существовавшим до обработки траекториям дефектов, может быть предложено как минимум два возможных объяснения. Во-первых, они могут быть порождены случайными флуктуациями электростатического потенциала. В случае, если в одном поперечном слое барьера, через который туннелируют электроны, находится несколько одинаковых энергетических уровней дефектов (локализованных состояний однотипных дефектов с равными энергиями связи), туннелирование через них будет происходить одновременно при одинаковом напряжении $V_{b}$ и суммарный ток будет состоять из равных парциальных вкладов всех этих каналов туннелирования. Наличие источника случайных флуктуаций потенциала в плоскости, параллельной слою с уровнями дефектов, приведет к локальным коррекциям их энергий такими флуктуациями относительно уровня Ферми по всему слою и, следовательно, изменению величин резонансных напряжений для каждого из уровней дефектов. Этот эффект проявлялся в туннелировании через донорные уровни, случайно расположенные в одном поперечном слое барьера с одинаковыми энергиями связей, в виде распадения единого туннельного канала с единым пороговым напряжением $V_{b}$ на парциальные локальные каналы туннелирования через отдельные примеси, т. е. мультиплицирования резонанса, как было продемонстрировано в работе [7], где источником случайных флуктуаций электростатического потенциала являлась обедненная смещением $V_{b}$ граница сильно легированного контактного слоя GaAs. C ростом температуры этот эффект приводит к уширению резонансного пика на величину, сопоставимую с амплитудой случайного потенциала. Отметим, что высокая поверхностная концентрация вакансий в графеновом слое после плазменной обработки значительно увеличивает вероятность геометрического совпадения их позиций с различными дефектами в барьерном слое и, следовательно, сильных локальных коррекций энергий связи разных типов барьерных дефектов. Во-вторых, по аналогии с полупроводниковыми гетеросистемами, где локальные коррекции порогов туннелирования через один тип де- фектов могут быть вызваны структурными неоднородностями гетерограниц, эквивалентными локальным изменениям эффективной толщины барьера, вызывающим изменения энергии связи, в ван-дер-ваальсовой гетеросистеме причиной подобных неоднородностей, вероятно, оказывается сильная структурная дефектность верхнего графенового слоя, возникшая после обработки плазмой. Вдобавок, несмотря на все предпринимавшиеся нами усилия, не исключена возможность внесения небольшого количества дефектов плазмой непосредственно в барьер. Последняя возможная причина не сопоставима с двумя предыдущими, а лишь может их дополнять, так как до обработки плазмой в образце уже было обнаружено количество дефектных резонансов, близкое к теоретическому пределу. Количественной оценки эффективной амплитуды случайного потенциала (по аналогии с данными работы [7]) в нашей ситуации сделать не представляется возможным, так как в работе [7] были предприняты специальные технологические усилия для того, чтобы реализовать гетеросистему, максимально приближенную к простой теоретической модели, когда существуют дефекты только одного типа, расположенные в одном поперечном слое. Контролируемого управления этими параметрами в ван-дер-ваальсовых гетероструктурах произвести невозможно принципиально. Однако, так как расстояние от источника флуктуаций потенциала до слоя с резонансными уровнями в нашем случае как минимум на порядок меньше, чем в работе [7], мы можем уверенно полагать, что эта величина в нашей ситуации значительно больше чем 10 мэВ, полученная из эксперимента в [7].

Таким образом, в данной работе исследовано резонансное туннелирование через локализованные состояния дефектов в барьерных слоях гетероструктур графен/ $h-\mathrm{BN} /$ графен с различной степенью совершенства слоев $h$-BN. Это позволило нам в рамках одного эксперимента исследовать проявления дефектных состояний в туннелировании от ситуации их полного отсутствия (в совершенной структуре) до нескольких десятков (после обработки плазмой) в запрещенной зоне $h$-BN и их связи с характеристическими зависимостями электростатической модели идеальной бездефектной гетероструктуры. Обнаружен эффект мультиплицирования туннельных резонансов через такие уровни, обусловленный влиянием высокой дефектности структуры соседнего слоя графена. В результате экспериментально показано, что ранее наблюдавшееся и оставшееся необъясненным многообразие дефектных резонансов может быть связано с влиянием случайных флуктуаций потенциала в контактных слоях графена.

\section{Финансирование работы}

Е.Е. Вдовин, Ю.Н. Ханин и С.В. Морозов благодарят за финансовую поддержку РНФ (грант № 17-12-01393), М.В. Григорьев выражает отдельную благодарность Программе президиума РАН „Изучение квантовых эффектов 
в веществе в конденсированном состоянии при сверхнизких температурах“. Авторы благодарят П.Л. Шабельникову за техническое содействие.

\section{Конфликт интересов}

Авторы заявляют, что у них нет конфликта интересов.

\section{Список литературы}

[1] U. Chandni, K. Watanabe, T. Taniguchi, J.P. Eisenstein. Nano Lett., 15 (11), 7329 (2015).

[2] U. Chandni, K. Watanabe, T. Taniguchi, J.P. Eisenstein. Nano Lett., 16 (12), 7982 (2016).

[3] Y. Liu, Zh. Tan, M. Kumar, T.S. Abhilash, G.-jun Liu, P. Hakonen. APL Materials, 6, 091102 (2018); doi: 10.1063/1.5042327.

[4] A. Ranjan, F.M. Puglisi, N. Raghavan, S.J. O’Shea, K. Shubhakar, P. Pavan, A. Padovani, L. Larcher, K.L. Pey. Appl. Phys. Lett., 112, 133505 (2018).

[5] M.T. Greenaway, E.E. Vdovin, D. Ghazaryan, A. Misra, A. Mishchenko, Y. Cao, Z. Wang, J.R. Wallbank, M. Holwill, Yu.N. Khanin, S.V. Morozov, K. Watanabe, T. Taniguchi, O. Makarovsky, T.M. Fromhold, A. Patané, A.K. Geim, V.I. Fal'ko, K.S. Novoselov, L. Eaves. Commun. Phys., 1, A94 (2018).

[6] N.R. Jungwirth, B. Calderon, Y. Ji, M.G. Spencer, M.E. Flatté, G.D. Fuchs. Nano Lett., 16, 6052 (2016).

[7] P. Mcdonnell, T.J. Foster, P.C. Main, L. Eaves, N. Mori, J.W. Sakai, M. Henini, G. Hill. Solid-State Electron., 40, 409 (1996).

[8] Ю.Н. Ханин, Е.Е. Вдовин, Ю.В. Дубровский. ФТП, 38 (4), 436 (2004).

[9] Е.Е. Вдовин, Ю.Н. Ханин. Письма ЖЭТФ, $108(9), 674$ (2018).

[10] L. Britnell, R.V. Gorbachev, R. Jalil, B.D. Belle, F. Schedin, A. Mishchenko, T. Georgiou, M.I. Katsnelson, L. Eaves, S.V. Morozov, N.M. Peres, J. Leist, A.K. Geim, K.S. Novoselov, L.A. Ponomarenko. Science, 335, 947 (2012).

[11] M. Zhu, D. Ghazaryan, S.-K. Son, C.R. Woods, A. Misra, L. He, T. Taniguchi, K. Watanabe, K. Novoselov, Y. Cao, A. Mishchenko. 2D Mater., 4, 011013 (2017).

[12] E.E. Vdovin, A. Mishchenko, M.T. Greenaway, M.J. Zhu, D. Ghazaryan, A. Misra, Y. Cao, S.V. Morozov, O. Makarovsky, T.M. Fromhold, A. Patané, G.J. Slotman, M.I. Katsnelson, A.K. Geim, K.S. Novoselov, L. Eaves. Phys. Rev. Lett., 116, 186603 (2016).

[13] D.C. Elias, R.R. Nair, T.M.G. Mohiuddin, S.V. Morozov, P. Blake, M.P. Halsall, A.C. Ferrari, D.W. Boukhvalov, M.I. Katsnelson, A.K. Geim, K.S. Novoselov. Science, 323, 610 (2009).

Редактор А.Н. Смирнов

\section{The role of structural imperfections of graphene in resonant tunneling through localized states in the $h$-BN barrier of van der Waals heterostructures}

\author{
M.V. Grigoriev' ${ }^{1}$ D.A. Ghazaryan ${ }^{2,3}$, E.E. Vdovin ${ }^{1}$, \\ Yu.N. Khanin ${ }^{1}$, S.V. Morozov ${ }^{1}$, K.S. Novoselov ${ }^{2,4}$ \\ ${ }^{1}$ Institute of Problems of Microelectronics \\ Technology and High Purity Materials, \\ Russian Academy of Sciences, \\ 142432 Chernogolovka, Moscow Region, Russia \\ ${ }^{2}$ School of Physics and Astronomy, \\ University of Manchester, \\ M13 9PL Manchester, United Kingdom \\ ${ }^{3}$ Department of Physics, National Research \\ University Higher School of Economics, \\ 105066 Moscow, Russia \\ ${ }^{4}$ Department of Material Science \& Engineering, \\ National University of Singapore, \\ 117575 Singapore, Republic of Singapore
}

Abstract Investigated resonant tunneling through defect levels in $h$-BN barrier van der Waals heterostructures. The effect of multiplication of tunnel resonances through these levels was found, due to the influence of a high degree of defectiveness of the structure of the neighboring layer of graphene, created by intentionally processing in plasma. Various mechanisms of such influence are discussed. 